\title{
1 Pourquoi et comment soigner plus précocement les troubles 2 psychotiques?
}

3

4 5

Why and how to treat psychosis earlier? Vincent Laprevote ${ }^{1,2,3}$, Ulrike Heitz ${ }^{2,}$, Paolo di Patrizio ${ }^{4}$, Erich Studerus ${ }^{2}$, Fabienne Ligier ${ }^{1,5}$,
Thomas Schwitzer $^{1,3}$, Raymund Schwan ${ }^{1,3,4}$, Anita Riecher-Rössler ${ }^{2}$

1. Centre Psychothérapique de Nancy, 1 rue du Dr Archambault, F-54 520 Laxou, France

2. Universität Basel, Universitäre Psychiatrische Kliniken, Zentrum für Gender Research und Früherkennung, Basel, Schweiz

3. EA 7298, INGRES, Université de Lorraine, Vandoeuvre-lès-Nancy F-54000, France

4. Faculté de Médecine, Université de Lorraine, Nancy F-54000, France

5. Université de Lorraine, Université Paris Descartes, EA 4360, APEMAC, Nancy, F-54000, France

\section{*auteur correspondant:}

Dr Vincent Laprévote, Centre Psychothérapique de Nancy, 1 rue du Dr Archambault, F-54 520 Laxou, France, v.laprevote@chu-nancy.fr

Conflits d'intérêt : Aucun.

Journal : La Presse Médicale

Rubrique : Mise au point

Mots-clefs : Psychose, Détection précoce, Intervention précoce, Médecine Générale

Keywords: Psychosis, Early detection, Early intervention, Generalist Practitioner

Nombre de caractères (espaces compris) : 28300 (Maximum 40 000)

Nombre de figures : 4

Nombre de tableaux : 1

Nombres de référence : 31 


\section{Pourquoi et comment soigner plus précocement les troubles}

psychotiques?

\section{Points essentiels}

- Les troubles psychotiques chroniques comme la schizophrénie sont des maladies qui débutent habituellement chez l'adulte jeune et qui peuvent être très invalidantes. Elles diminuent l'espérance de vie de 22 ans en moyenne.

- Les modèles actuels décrivent une installation progressive des troubles psychotiques. Ceuxci peuvent être précédés d'un état mental à risque de psychose caractérisé par des symptômes désormais bien répertoriés.

- Dans un système de soins tenant compte uniquement du premier épisode psychotique, la durée de maladie non traitée (DMNT) peut atteindre plusieurs années. Or cette DMNT a un impact direct sur le pronostic de la maladie.

- Les recommandations internationales actuelles préconisent de réduire la DMNT en détectant et en intervenant précocement dans les états mentaux à risque de psychose. Cela permet également au patient de s'insérer dans les soins dans un moment où il est pleinement en état d'y consentir et d'y adhérer.

- Le médecin généraliste est un acteur capital de la détection précoce des troubles psychotiques. La détection des états mentaux à risque de psychose en cabinet de médecine générale peut être réalisée par des moyens simples et standardisés que nous décrivons ici.

- De nombreux pays dans le monde ont développé des centres de détection et d'intervention précoce pour les états mentaux à risque de psychose. Il est démontré que ces interventions diminuent nettement le risque de transition vers une psychose constituée et améliorent le pronostic.

- Les données récentes concernant ces mesures de détection et d'intervention précoce constituent une avancée majeure dans la pratique de la psychiatrie. Il est désormais nécessaire de développer largement de telles mesures en France. 
89 Why and how to treat psychosis earlier in France?

90

91

92

93

94

95

96

97

98

99

100

101

102

103

104

105

106

107

108

109

110

111

112

113

114

115

116

117

118

119

120

121

122

123

124

125

\section{Key-points}

- Chronic psychosis, as for instance schizophrenia, usually begins in young adulthood and may cause severe disability. It causes a mean loss of life expectancy of 22 years

- Actual models of psychosis do not trace the beginning of psychosis to the first franc psychotic episode only, but to earlier symptoms.

- In a classical health system only considering the first psychotic episode, the mean duration of untreated illness (DUI) can last several years. Yet this DUI has a direct impact on the prognosis of the disease.

- Actual international recommendations prescribe to early detect and treat at risk mental states of psychosis, thus reducing DUI. Such an attitude also helps the patient to integrate care in a moment where she/he is fully in condition to consent and to adhere.

- Generalist practitioners are crucial actors of early detection. We describe here simple and standardized tools helping early detection of high risk mental states of psychosis in primary care and the appropriate attitude to do it properly.

- Numerous countries have developed early detection and treatment centers for psychosis. It has been established that such interventions clearly decrease the risk of transition towards chronic psychosis and improve the prognosis.

- These recent data about early detection and intervention in psychosis are a major step forward in psychiatry practice. It is now necessary to largely develop such actions in France. 
128 Les troubles psychotiques chroniques, dont la schizophrénie est la plus fréquente, peuvent

129 constituer une épreuve très lourde pour les patients et leurs familles. Actuellement la prévalence de

130 la schizophrénie est estimée à 0.5 à $1 \%$ de la population, soit un risque individuel de $0.7 \%$ sur la vie

131 entière [1,2]. Ces troubles sont caractérisés par la survenue chez l'adulte jeune de symptômes dits

132 positifs, regroupant les hallucinations et le délire, de symptômes négatifs comme l'apragmatisme, la 133 pauvreté du langage ou le retrait social, et de symptômes de désorganisation, correspondant à une 134 perte de l'unité et de la cohérence des pensées, du discours, des affects ou du comportement. Les traitements reconnus de ces troubles psychotiques chroniques associent une pharmacothérapie basée sur la prescription de médicaments antipsychotiques à des moyens de psychothérapie et à la 137 réhabilitation psychosociale du patient.

139 L'amélioration de ces soins a permis une nette amélioration du devenir des patients au cours des 140 dernières décennies [3], mais les troubles psychotiques peuvent rester d'une grande gravité. Si 10 à $14120 \%$ des patients connaîtront une récupération complète et durable de leurs capacités, d'autres 142 patients auront une récupération seulement partielle des troubles, voire devront affronter des 143 symptômes persistants [4]. Du fait du risque de permanence des symptômes et de la longue durée de 144 la maladie, la schizophrénie a été classée par l'OMS parmi les 10 causes les plus importantes de 145 handicap [5]. Mais surtout ces troubles sont marqués par une importante perte d'espérance de vie, 146 estimée à 22 ans pour la schizophrénie [6]. Il faut souligner que les principales causes de mortalité 147 précoce de la population générale sont retrouvées dans la schizophrénie et que toutes sont 148 augmentées très significativement [7]. Parmi ces causes de mortalité précoce, le suicide tient une 149 place importante puisque le risque sur une vie entière de mourir par suicide est 12 fois supérieur à la 150 population générale [7]. Pour toutes ces raisons, l'ensemble des acteurs de santé sont concernés par 151 cette maladie grave, et en premier lieu le médecin généraliste qui est un recours permanent dans ces 
152 situations de longue durée, mais qui est aussi souvent l'un des premiers à constater le début des

153 troubles, bien avant les acteurs spécialisés.

154

155 Étant donné que les troubles psychotiques surviennent à un âge jeune, lorsque les patients sont en 156 pleine réalisation de leur construction personnelle et professionnelle, et compte tenu de certains

157 symptômes comme le retrait social, ces troubles mettent gravement en péril l'insertion sociale et 158 professionnelle. Leur survenue à un jeune âge peut donc avoir des conséquences sur de très 159 nombreuses années après la survenue des premiers symptômes.

160

161 A côté de ces conséquences personnelles dramatiques, les seuls soins des troubles psychotiques 162 représentent un coût moyen annuel de $23929 €$ pour une personne en France [8]. Plus précisément, 163 l'ensemble des coûts annuels pour la société a été estimé au Royaume Uni à 6.7 milliards de livres 164 en 2004-2005 dont entre autres 2 milliards pour les soins et 4.7 milliards de coûts indirects pour la 165 société [9]. 
De manière conventionnelle, le traitement des troubles psychotiques a longtemps été considéré comme devant débuter au premier épisode psychotique constitué, sans prêter attention à la période précédant ce premier épisode. Or la littérature a largement démontré qu'il existait souvent un long délai entre l'apparition du premier épisode psychotique et l'instauration d'un traitement médical efficace. Dans un système de soins conventionnel, le retard diagnostique et d'accès aux soins engendre une durée de psychose non traitée (DPNT) estimée à 1 à 2 ans, ce qui constitue un retard au traitement considérable [10,11]. Avant l'éclosion franche des symptômes psychotiques (voir la figure 1), cette DPNT est souvent précédée de symptômes prodromaux, non spécifiques mais pouvant être identifiés précocement ce qui rajoute à cette durée de psychose non traitée, une durée totale de maladie non traitée (DMNT) plus longue encore [12].

De fait, la littérature actuelle fait émerger un modèle très progressif concernant le début de la psychose (voir en résumé la figure 2, pour une revue de littérature voir Fusar Poli [13] ou Krebs [14]). Cette progressivité permet, selon un certain nombre de critères diagnostiques, d'identifier des états mentaux à risque de psychose. Deux types de critères ont coexisté dans la littérature pour décrire ces états à risque : les symptômes de base et les critères "ultra-high-risk” (UHR). Les symptômes de base ont été les premiers à avoir été décrits historiquement par les équipes allemandes, en particulier sur la base des travaux phénoménologiques de Gert Huber (GROSS G.,

188 HUBER G. (1985), « Psychopathology of basic stages of schizophrenia in view of formal thought 189 disturbances », Psychopathology, 18(2-3):115-25.) [15]. Ces symptômes sont des perturbations 190 subjectives expérimentées dans différents domaines, comme la perception, le contenu de la pensée, 191 le langage, l'attention, et qui sont distincts des symptômes psychotiques classiques car ils ne 
192 contiennent pas de perte franche de contact avec la réalité et le patient lui-même se les représente

193 comme anormaux. Les critères $U H R$ sont les plus utilisés dans la littérature [16]. Leur application

194 requiert l'apparition d'un ou plusieurs des critères suivants : l'apparition de symptômes positifs

195 atténués (SPA) qui sont d'authentiques symptômes psychotiques durables mais dont l'intensité

196 n'atteint pas un niveau suffisant pour qualifier un épisode psychotique selon les critères du DSM ;

197 l'apparition souvent plus tardive de symptômes psychotiques limités intermittents (SPLI) et brefs (en

198 anglais Brief Limited Intermittent Psychotic Symptoms, BLIPS) qui sont des expériences 199 psychotiques franches mais fugaces disparaissant en moins d'une semaine ; la présence d'une 200 vulnérabilité génétique identifiée dans les antécédents familiaux associée à un déclin marqué du 201 fonctionnement psychosocial ; la présence de symptômes prodromaux non spécifiques.

203 Il faut noter que les définitions des symptômes de base et des critères $U H R$ ne les opposent pas mais 204 les rendent complémentaires. Ainsi, la compréhension actuelle de la maladie les associe en plaçant 205 les symptômes de base dans une phase précoce d'état à risque de psychose et les SPA et les SPLI 206 dans une phase tardive d'état à risque de psychose [13]. La figure 2 reprend l'articulation de ces 207 différents symptômes dans le développement de l'état à risque de psychose.

209 Pour les personnes présentant un état mental à risque de psychose, il s'agit donc de veiller au risque 210 de transition vers la psychose. Dans la littérature un éventail de différents critères a été employé 211 pour définir cette transition. Classiquement ces critères se rapprochent de ceux définis par Yung et 212 al [17] : la survenue d'au moins un symptôme positif franc plusieurs fois par semaine pendant plus 213 d'une semaine. Toutes les personnes qui présentent un état mental à risque de psychose ne feront 214 pas cette transition vers une psychose constituée. On retrouve dans les cohortes qui suivent les états 215 mentaux à risque un taux de transition relativement constant, compris entre $18 \%$ à 6 mois de suivi et $21636 \%$ après 3 ans de suivi, malgré les disparités méthodologiques et de populations entre les 217 différentes études [18]. 


\section{Pourquoi détecter et intervenir précocement ?}

225 Idéalement, repérer et intervenir auprès d'un patient à risque avant la transition psychotique a pour

226 but de prévenir la transition psychotique, ou au moins la retarder. Comme on le verra plus loin, certains dispositifs arrivent effectivement à diminuer ce taux de transition et donc à prévenir les troubles psychotiques [19]. Mais même dans les cas où la transition se produit, on sait désormais qu'en diminuant la DMNT [15], avoir été mis en contact avec les soins dès le stade d'état mental à risque de psychose est important pour le pronostic de la maladie, car plus une psychose est traitée tardivement, plus la probabilité de rémission des symptômes positifs diminue, plus la sévérité générale de la maladie s'aggrave, et plus le fonctionnement général et le taux de retour à l'emploi sont diminués [20]. Une intervention précoce a donc pour but de réduire la DMNT, ce qui pourrait 234 améliorer le pronostic.

La qualité de l'alliance thérapeutique est primordiale dans le suivi d'un trouble psychotique. Or, attendre le moment de l'éclosion de l'ensemble du tableau psychotique pour débuter une démarche thérapeutique expose le patient à accéder aux soins dans les pires conditions : à un moment où sa capacité d'adhésion aux soins est altérée par la perte de contact avec la réalité. L'instauration des

240 soins peut alors nécessiter jusqu'à une mesure de soins sans consentement qui peut elle-même gêner

241 le processus d'adhésion aux soins. Intervenir avant l'éclosion d'un trouble psychotique franc permet

242 de délivrer les informations à un moment où la personne est encore en état d'adhérer à une 243 proposition de soins. Par ailleurs, une longue DMNT risque de dégrader des liens sociaux 
244 essentiels, qui pourront encore être distendus en cas de trouble psychotique franc, y compris si

245 celui-ci nécessite une hospitalisation longue. Intervenir avant la crise permet également de préserver

246 au mieux le lien social.

247

248 Cette intervention précoce est désormais recommandée par de nombreuses sociétés psychiatriques

249 nationales ou internationales, comme l'Institut National Britannique pour la Santé et l'Excellence 250 des Soins [21] ou l'Association européenne de Psychiatrie [19] Cependant, les soins psychiatriques 251 en France sont encore peu structurés pour permettre la détection et l'intervention précoce. Ce fait 252 offre un contraste saisissant avec le Royaume Uni où de nombreux centres spécifiques sont mis en 253 place, permettant aux acteurs de première ligne d'adresser rapidement leurs patients. 


\section{Détecter plus précocement les psychoses en France: rôle des soins primaires}

257 L'information et la vigilance des acteurs de soins primaires sont essentielles à la détection des états 258 mentaux à risque. Les meilleurs lieux de détection précoce sont les lieux non spécialisés, peu

259 stigmatisants. Parmi les différentes ressources disponibles pour le patient, le cabinet du médecin 260 généraliste peut donc constituer un lieu privilégié de détection et d'orientation des états mentaux à 261 risque. S'il est évident que la détection d'une psychose débutante ne constitue pas une tâche 262 quotidienne pour un médecin généraliste, recevoir un tel patient n'est pas un évènement 263 impossible : une enquête menée chez les médecins généralistes irlandais a montré que $71 \%$ des 264 participants recevaient 3 à 5 patients par an pour une psychose débutante [22]. Notons d'ailleurs que 265 le fait d'avoir un médecin traitant désigné améliore les conditions de soins lors d'un premier 266 épisode psychotique [23].

268 En médecine générale, le cas d'un patient jeune (moins de 30 ans chez un homme, moins de 40 ans 269 chez une femme) qui consulte pour des symptômes psychiatriques atypiques est une situation qui 270 peut interpeller quant au risque de psychose. Il est désormais établi que la consommation régulière 271 de cannabis est un facteur de risque de troubles psychotiques. La présence d'une telle 272 consommation ne doit donc pas faussement rassurer un médecin lors de la survenue des symptômes 273 décrits ci-dessous mais bien constituer un facteur d'inquiétude supplémentaire.

275 D’un point de vue clinique, les symptômes qui peuvent retenir l'attention sont assez diversifiés. En 276 reprenant le modèle développé par Fusar Poli et al. [13] (voir figure 2) on peut retrouver dans les 277 années qui précèdent l'éclosion du premier épisode psychotique franc des signes avant-coureurs 278 précoces, non spécifiques, qui constituent les symptômes de base. Ils sont immédiatement reconnus 279 par le patient lui-même comme dysfonctionnels et ne sont pas attribués à l'environnement comme 280 peuvent l'être les symptômes psychotiques positifs. Il est important de souligner qu'ils sont en 
rupture avec le fonctionnement habituel du patient. Il s'agit ainsi de modifications récentes dans la manière de penser, de parler ou dans la perception de l'environnement. Concernant les processus de

283 pensée, on peut ainsi retrouver des interférences de pensée, des persévérations ou des blocages de pensée, ainsi que des difficultés à manier les raisonnements abstraits, des troubles attentionnels. De subtils troubles de l'émission ou de la perception du langage peuvent également être présents. On peut aussi retrouver des difficultés à discriminer le produit de l'imagination de la perception de la réalité, de la déréalisation ou des idées de références fugaces, c'est-à-dire que des significations personnels sont données à des événements anodins de l'environnement. Enfin des perturbations sensorielles fines peuvent survenir comme une hypersensibilité à la lumière ou aux sons [15].

291 A un stade plus avancé, on peut observer des symptômes psychotiques atténués. Il s'agit de 292 symptômes psychotiques à bas bruit, qui seront des précurseurs de délire ou d'hallucinations. Ceux-

293 ci n'atteignent cependant pas une intensité suffisante pour être des symptômes psychotiques positifs 294 selon les classifications : un certain niveau de conscience des symptômes peut ainsi être préservé ainsi qu'une capacité à s'adapter à ces symptômes. Certains patients peuvent se sentir «comme dans un film», se sentir étranger à leur propre corps, se sentir observés, suivis ou menacés. Cependant, on ne retrouve pas à ce stade de conviction inébranlable, qui serait un critère de délire constitué. Certains patients peuvent également ressentir des modifications des perceptions, sans toutefois que les critères d'hallucinations, en tant que perception sans objet, ne soient pleinement remplis. Des hallucinations partielles et temporaires peuvent également se produire, comme le fait de se sentir appelé brièvement par son nom. [16]

303 On peut également observer à ce stade des symptômes psychotiques limités intermittents (SPLI) : les

304 idées délirantes et les hallucinations sont franches mais ne sont présentes que pour une durée courte 305 et temporaire (de quelques minutes à une semaine, avec rémission spontanée). Le stade ultérieur 306 sera la première décompensation psychotique marquée par des symptômes psychotiques aigus 
persistants comme le délire, les hallucinations ou la désorganisation de la pensée. [16]

309 Évidemment, il ne s'agit pas d'envisager une évaluation spécialisée des états mentaux à risque dans

310 le cabinet de médecine générale. Dans le contexte très contraint de la médecine générale, une

311 évaluation rapide est d'ailleurs plus réaliste. Il s'agit donc avant tout de pouvoir dépister et adresser

312 les patients. Dans ce but des outils standardisés ont été développés pour faciliter ce dépistage. La

313 figure 3 reprend une traduction et les modalités d'utilisation du questionnaire d'auto-évaluation de

314 Bâle (Suisse) pour le risque de psychose [24]. Ce test est un auto-questionnaire qui peut être réalisé

315 par le patient en salle d'attente ou à domicile et qui peut ensuite servir de base à une discussion avec

316 le médecin généraliste.

Insérer ici la figure 3

320 Comment l'acteur de soins primaires doit il se comporter et réagir lors de la découverte d'un état mental à risque de psychose ? Il s'agit avant tout d'adapter son attitude à la symptomatologie. Dans

322 le contexte de méfiance qui peut accompagner cet état mental, il est utile d'instaurer une prise de 323 contact progressive et prudente, permettant au patient de se mettre en confiance. De même, il peut

324 être nécessaire de tenir compte des capacités réduites d'écoute et de compréhension du patient : il

325 faudra alors se mettre à son écoute, respecter son point de vue, adopter un langage simple, être 326 progressif dans la récolte des informations. L'évaluation du risque suicidaire est importante dans ce 327 contexte. Une fois l'état constaté médicalement, l'explication de la conduite à tenir doit également 328 être prudente et progressive : il est ainsi utile de prendre en compte la peur et le refus potentiel 329 d'une démarche psychiatrique tout en insistant sur sa nécessité. Il convient dans tous les cas de se 330 comporter de manière claire, transparente et loyale et, notamment lorsque l'on se situe dans la 331 période à risque avant l'éclosion des symptômes psychotiques, d'éviter au maximum les mesures 332 coercitives. Intégrer les proches aux différents stades d'évaluation et de soins permettra 
333 d'augmenter l'efficacité de la démarche. Enfin, il sera bien souvent indispensable de prendre au

334 sérieux l'anxiété générée par les troubles, ce qui nécessitera parfois un traitement médicamenteux,

335 mais il convient d'éviter au maximum la prescription immédiate d'antipsychotiques pour privilégier

336 plutôt les traitements symptomatiques brefs comme des benzodiazépines sur une courte durée.

338 Si un état mental à risque de psychose est constaté, il est nécessaire de convaincre le patient de se

339 présenter rapidement dans un centre adapté. Dans le contexte français actuel, il s'agira de l'adresser

340 vers un Centre Médico-Psychologique ou vers un psychiatre libéral, mais le chapitre suivant décrit

341 les centres spécialisés qui ont été mis en place dans de nombreux pays et dont on peut souhaiter

342 qu'ils voient prochainement le jour en France. Du point de vue du médecin généraliste, il est

343 d'ailleurs frappant de constater que, dans un contexte de psychose débutante, ceux-ci privilégieront

344 un service facile d'accès, flexible, peu stigmatisant, et qui communique sur ses évaluations [25]. 


\section{Evaluer et intervenir : les centres de détection et d'intervention précoce}

347 La nécessité de fournir une évaluation et des soins spécifiques pour les états mentaux à risque de 348 psychose a conduit à la création de centres spécialisés dans le repérage et l'intervention précoce.

349 Les premiers centres ont été développés dans les années 1980-1990 en Australie par l'équipe de 350 Patrick Mc Gorry [26]. De nombreuses équipes dans le monde ont repris ce modèle et l'ont adapté à 351 leur contexte, comme par exemple au Royaume Uni, en Suisse, en Allemagne, au Canada, aux Etats

352 Unis. Certains états se sont même dotés de programmes gouvernementaux afin de développer un 353 maillage territorial, comme c'est le cas au Royaume Uni, en Australie ou au Canada. S'il existe 354 évidemment des différences culturelles selon les pays d'implantation de ces programmes, il existe 355 de nombreuses similitudes dues aux caractéristiques cliniques des états mentaux à risque de 356 psychose.

358 Les soins développés pour les états mentaux à risque sont essentiellement des soins ambulatoires. Il 359 est en effet absolument primordial de privilégier au maximum les liens sociaux et familiaux du 360 patient, alors même que les symptômes menacent ces liens. De nombreux auteurs insistent sur le 361 fait que ces centres doivent être spécifiques de la classe d'âge visée [21], notamment en proposant 362 un environnement et des pratiques adaptées à l'âge, notion schématiquement décrite comme « youth 363 friendly» [26]. Ce point est essentiel pour favoriser l'adhésion du patient à la démarche qui sera 364 proposée. Dans le même but, un environnement non stigmatisant est souvent choisi (en plaçant par 365 exemple le centre à distance de l'hôpital psychiatrique) et la plupart des centres se montrent très 366 faciles d'accès et très réactifs, proposant des rendez-vous rapides.

368 Il s'agit de centres pluridisciplinaires, dans lesquels peuvent collaborer des médecins psychiatres, 369 des psychologues, des infirmiers, des travailleurs sociaux. Classiquement, ces centres fonctionnent 370 sur le modèle du case management : un professionnel (généralement non médecin) est chargé de la 
371 coordination de toutes les mesures proposées au patient. Il est son référent et le voit donc plus

372 fréquemment que tout autre professionnel. Il maintient la continuité du suivi, sa cohérence et facilite

373 l'établissement d'une relation de confiance. Ce rôle peut être comparé au rôle de référent hospitalier

374 pour la continuité des soins décrit dans le système de santé belge (La fonction de référent hospitalier

375 pour la continuité des soins. L'HOPITAL BELGE, n² 232, 1998/01-03, pages 48-52, réf.bibl., BEL

376 DEJACE (A.), GOSSET (C.), RONDAL (P.), et al.). Un case manager a donc peu de patients dans

377 sa file active, environ 10 à 30 [27]. Le psychiatre a bien plus de patients en file active car il

378 intervient essentiellement sur les aspects médicaux des soins et assure généralement une supervision

379 globale du centre.

L'une des fonctions essentielles de ce type de centres est d'abord de faire une évaluation initiale complète, et de renouveler l'évaluation régulièrement. Selon les équipes différents moyens sont utilisés pour effectuer l'évaluation initiale des états mentaux à risque de psychose. Le tableau I reprend les différents outils disponibles dans la littérature internationale et précise ceux qui sont traduits en français. Une évaluation initiale des performances neurocognitives, et la réalisation d'un EEG et d'une IRM de base sont également préconisées. La figure 4 reprend à titre d'exemple

387 l'ensemble des évaluations réalisées lors du premier contact au centre de détection précoce de Bâle 388 (Suisse).

395 Lorsque l'état mental à risque de psychose est constaté, des mesures thérapeutiques spécifiques sont 396 mises en œuvre. Ainsi le case manager peut organiser avec le patient des sessions d'information et 
psychoéducation du patient et des proches, d'entretiens motivationnels, de programmes

398 d'entraînement psychologique comme la gestion du stress, de thérapies cognitivo-

399 comportementales, de remédiation cognitive. L'utilisation de médicaments reste centrée sur les

400 aspects symptomatiques, par exemple à visée anxiolytique. Il a été démontré que la prescription

401 systématique d'antipsychotiques n'aidait pas à améliorer le devenir des états à risque de psychose

402 en l'absence de symptômes positifs [19]. En revanche, en cas de survenue de symptômes positifs

403 constitués, l'utilisation d'antipsychotiques ne doit pas être retardée mais débuter à des doses faibles,

404 et en n'employant que des augmentations progressives si elles sont nécessaires.

406 Nombre d'essais cliniques ont évalué l'efficacité des interventions réalisées dans ces centres, ce qui

407 a donné lieu à plusieurs méta-analyses [19,28]. La méta-analyse la plus récente dénombrait 15

408 essais cliniques comparatifs et randomisés et concluait à une efficacité significative de ces

409 dispositifs, permettant une réduction du risque de transition vers la psychose de $64 \%$ à 6 mois

410 (nombre de sujets à traiter $=15$ ) et de $59 \%$ à 18 mois (nombre de sujets à traiter $=13$ ). Ces résultats

411 remarquables font désormais de ces centres un outil important de la réponse sociétale aux troubles

412 psychotiques. On ne peut que déplorer l'absence de structuration d'une telle offre en France. Les

413 importants résultats scientifiques relevés à Paris dans le programme C'JAAD (Centre d'Evaluation

414 pour les Jeunes Adultes et Adolescents) ont joué un rôle pilote dans notre pays [29]. A l'heure de

415 l'écriture de cet article, plusieurs autres centres de soins sont en cours de constitution en France. 


\section{Conclusion}

417

418 La détection et l'intervention précoces pour les troubles psychotiques constituent actuellement un

419 tournant majeur dans la prise en charge de ces troubles. Il est désormais démontré que ces

420 interventions sont efficaces et particulièrement respectueuses des individus. Elles impliquent la

421 création de centres ambulatoires spécialisés qui manquent encore largement en France. Mais il est

422 important de noter que de tels centres ne peuvent fonctionner que si les acteurs de premier plan sont

423 en position de pouvoir repérer les patients à risque. Cela implique particulièrement les médecins

424 généralistes qui peuvent désormais effectuer ce repérage grâce à des outils faciles à utiliser, 425 disponibles, traduits en français et simples à intégrer dans un dispositif médical. 
[1] Saha S, Chant D, Welham J, McGrath J. A systematic review of the prevalence of schizophrenia. PLoS Med 2005;2:e141. doi:10.1371/journal.pmed.0020141.

[2] McGrath J, Saha S, Chant D, Welham J. Schizophrenia: a concise overview of incidence, prevalence, and mortality. Epidemiol Rev 2008;30:67-76. doi:10.1093/epirev/mxn001.

[3] Hegarty JD, Baldessarini RJ, Tohen M, Waternaux C, Oepen G. One hundred years of schizophrenia: a meta-analysis of the outcome literature. Am J Psychiatry 1994;151:1409-16. doi:10.1176/ajp.151.10.1409.

[4] Jääskeläinen E, Juola P, Hirvonen N, McGrath JJ, Saha S, Isohanni M, et al. A systematic review and meta-analysis of recovery in schizophrenia. Schizophr Bull 2013;39:1296-306. doi:10.1093/schbul/sbs130.

[5] Organization WH. The world health report 2001: mental health: new understanding new hope. World Health Organization; 2001.

[6] Tiihonen J, Lönnqvist J, Wahlbeck K, Klaukka T, Niskanen L, Tanskanen A, et al. 11-year follow-up of mortality in patients with schizophrenia: a population-based cohort study (FIN11 study). Lancet Lond Engl 2009;374:620-7. doi:10.1016/S0140-6736(09)60742-X.

[7] Saha S, Chant D, McGrath J. A systematic review of mortality in schizophrenia: is the differential mortality gap worsening over time? Arch Gen Psychiatry 2007;64:1123-31. doi:10.1001/archpsyc.64.10.1123.

[8] Raymond S, Martin M, Netillard C, Mejat-Alder E, Woronoff-Lemsi M, Tissot E. Schizophrénie : évaluation du coût de la prise en charge. J Gest Déconomie Médicales 2009; Volume 27:421-33.

[9] Mangalore R, Knapp M. Cost of schizophrenia in England. J Ment Health Policy Econ 2007;10:23-41.

[10] Loebel AD, Lieberman JA, Alvir JM, Mayerhoff DI, Geisler SH, Szymanski SR. Duration of psychosis and outcome in first-episode schizophrenia. Am J Psychiatry 1992;149:1183-8. doi:10.1176/ajp.149.9.1183.

[11] White C, Stirling J, Hopkins R, Morris J, Montague L, Tantam D, et al. Predictors of 10-year outcome of first-episode psychosis. Psychol Med 2009;39:1447-56. doi:10.1017/S003329170800514X.

[12] Riecher-Rössler A, Gschwandtner U, Borgwardt S, Aston J, Pflüger M, Rössler W. Early detection and treatment of schizophrenia: how early? Acta Psychiatr Scand Suppl 2006:73-80. doi:10.1111/j.1600-0447.2005.00722.x.

[13] Fusar-Poli P, Borgwardt S, Bechdolf A, Addington J, Riecher-Rössler A, Schultze-Lutter F, et al. The psychosis high-risk state: a comprehensive state-of-the-art review. JAMA Psychiatry 2013;70:107-20. doi:10.1001/jamapsychiatry.2013.269.

[14] Krebs M-O, CPNLF. Signes précoces de schizophrénie. Dunod; 2015.

[15] Schultze-Lutter F. Subjective symptoms of schizophrenia in research and the clinic: the basic symptom concept. Schizophr Bull 2009;35:5-8. doi:10.1093/schbul/sbn139.

[16] Schultze-Lutter F, Michel C, Schmidt SJ, Schimmelmann BG, Maric NP, Salokangas RKR, et al. EPA guidance on the early detection of clinical high risk states of psychoses. Eur Psychiatry J Assoc Eur Psychiatr 2015;30:405-16. doi:10.1016/j.eurpsy.2015.01.010.

[17] Yung AR, Phillips LJ, McGorry PD, McFarlane CA, Francey S, Harrigan S, et al. Prediction of psychosis. A step towards indicated prevention of schizophrenia. Br J Psychiatry Suppl 1998;172:14-20.

[18] Fusar-Poli P, Bonoldi I, Yung AR, Borgwardt S, Kempton MJ, Valmaggia L, et al. Predicting psychosis: meta-analysis of transition outcomes in individuals at high clinical risk. Arch Gen 
Psychiatry 2012;69:220-9. doi:10.1001/archgenpsychiatry.2011.1472.

[19] Schmidt SJ, Schultze-Lutter F, Schimmelmann BG, Maric NP, Salokangas RKR, RiecherRössler A, et al. EPA guidance on the early intervention in clinical high risk states of psychoses. Eur Psychiatry J Assoc Eur Psychiatr 2015;30:388-404.

doi:10.1016/j.eurpsy.2015.01.013.

[20] Schimmelmann BG, Huber CG, Lambert M, Cotton S, McGorry PD, Conus P. Impact of duration of untreated psychosis on pre-treatment, baseline, and outcome characteristics in an epidemiological first-episode psychosis cohort. J Psychiatr Res 2008;42:982-90. doi:10.1016/j.jpsychires.2007.12.001.

[21] NICE. Psychosis and schizophrenia in adults - NICE Pathways 2014. http://pathways.nice.org.uk/pathways/psychosis-andschizophrenia\#path=view\%3A/pathways/psychosis-and-schizophrenia/psychosis-andschizophrenia-in-adults.xml\&content=view-node\%3Anodes-early-intervention-for-firstepisode-psychosis (accessed December 2, 2015).

[22] Gavin B, Cullen W, O’Donoghue B, Ascencio-Lane JC, Bury G, O'Callaghan E. Schizophrenia in general practice: a national survey of general practitioners in Ireland. Ir J Med Sci 2005;174:38-42.

[23] Cole E, Leavey G, King M, Johnson-Sabine E, Hoar A. Pathways to care for patients with a first episode of psychosis. A comparison of ethnic groups. Br J Psychiatry J Ment Sci 1995;167:770-6.

[24] Kammermann J, Stieglitz R-D, Riecher-Rössler A. "Selbstscreen-Prodrom” - ein Selbstbeurteilungsinstrument zur Früherkennung von psychischen Erkrankungen und Psychosen. Fortschritte Neurol Psychiatr 2009; Vol. 77:S. 278-84.

[25] Gavin B, Cullen W, Foley S, McWilliams S, Turner N, O’Callaghan E, et al. Integrating primary care and early intervention in psychosis services: a general practitioner perspective. Early Interv Psychiatry 2008;2:103-7. doi:10.1111/j.1751-7893.2008.00065.x.

[26] McGorry PD. Early intervention in psychosis: obvious, effective, overdue. J Nerv Ment Dis 2015;203:310-8. doi:10.1097/NMD.0000000000000284.

[27] Baumann PS, Crespi S, Marion-Veyron R, Solida A, Thonney J, Favrod J, et al. Treatment and early intervention in psychosis program (TIPP-Lausanne): Implementation of an early intervention programme for psychosis in Switzerland. Early Interv Psychiatry 2013;7:322-8. doi:10.1111/eip.12037.

[28] Marshall M, Rathbone J. Early intervention for psychosis. Cochrane Database Syst Rev 2011:CD004718. doi:10.1002/14651858.CD004718.pub3.

[29] Krebs M-O, Magaud E, Willard D, Elkhazen C, Chauchot F, Gut A, et al. [Assessment of mental states at risk of psychotic transition: validation of the French version of the CAARMS]. L'Encéphale 2014;40:447-56. doi:10.1016/j.encep.2013.12.003. 


\section{Remerciements :}

Les auteurs remercient l'Association des Médecins du Centre Psychothérapique de Nancy qui a soutenu cette collaboration franco-suisse. 
429 Légendes des figures et tableaux

431 Figure 1 : L'apparition des symptômes psychotiques précède souvent le traitement, constituant une

432 durée de psychose non traitée de un à deux ans. Ces symptômes psychotiques peuvent être eux 433 mêmes précédés de symptômes prodromaux non spécifiques.

435 Figure 2 : Modèle progressif de développement des troubles psychotiques, d'après Fusar Poli [13] 436 et d'après Krebs [14] pour la traduction française.

438 Figure 3: Questionnaire d'auto-évaluation de Bâle pour le risque de psychose (traduction 439 française, d'après [24])

441 Tableau I: Principales échelles permettant l'évaluation initiale du risque de psychose.

443 Figure 4 : Evaluations effectuées chez une personne présentant un état mental à risque au Centre 444 de Reconnaissance Précoce des Psychoses de Bâle (Suisse). Cette liste est citée à titre d'exemple.

445 Elle est particulièrement exhaustive du fait de la vocation de recherche du centre. 